\title{
Investigating the metabolism of glucoraphanin by the human gut microbiota using ex vivo culturing methods
}

\author{
L. Kellingray ${ }^{1}$, S. Saha ${ }^{1}$, J. Doleman ${ }^{1}$, A. Narbad ${ }^{2}$ and R. Mithen ${ }^{1}$ \\ ${ }^{1}$ Food and Health, Institute of Food Research, Norwich, NR4 $7 U A$ and ${ }^{2}$ Gut Health and Safety, Institute of Food \\ Research, Norwich, NR4 $7 U A$
}

Glucosinolates are sulfur-rich compounds found in cruciferous vegetables, such as broccoli, and have been linked to a reduced risk of cancer and cardiovascular diseases ${ }^{(1,2)}$. Studies suggest that bioactivity is due to the glucosinolate hydrolysis products, isothiocyanates (ITCs), which are generated from glucosinolates by the plant enzyme myrosinase, or myrosinase-like enzymes found in human gut bacteria $^{(3)}$. To examine the effects of glucoraphanin (GR), the predominant glucosinolate found in broccoli, on the human gut microbiota, an experimental model was set-up in which human faecal bacteria were exposed to a repeated dose of GR for seven 12-hour cycles. A broccoli leachate was added to a bacterial media designed to mimic the nutrients that the human gut microflora would encounter in the human gut, and used to culture the human faecal bacteria in anaerobic conditions at $37^{\circ} \mathrm{C}$. A fresh broccoli leachate media was spiked after each 12-hour cycle and this was continued for seven cycles. The faecal bacteria converted a large portion of the GR to glucoerucin via a bacterial-mediated reduction reaction (figures 1 and 2), and metabolised a small amount of GR to form the ITC sulforaphane (SF), as well as SF nitrile and SF conjugates.

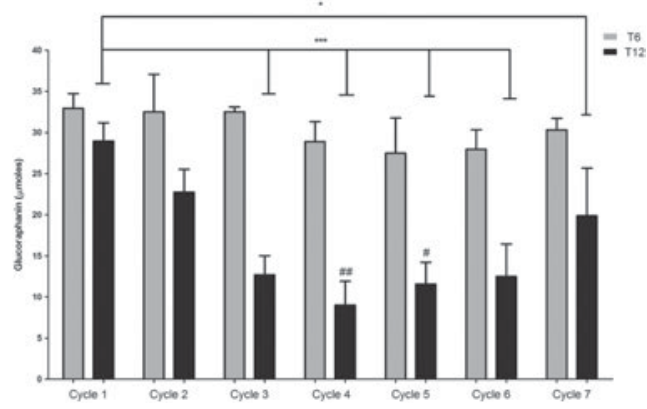

Fig. 1. Decrease in GR across seven 12 -hour cycles.

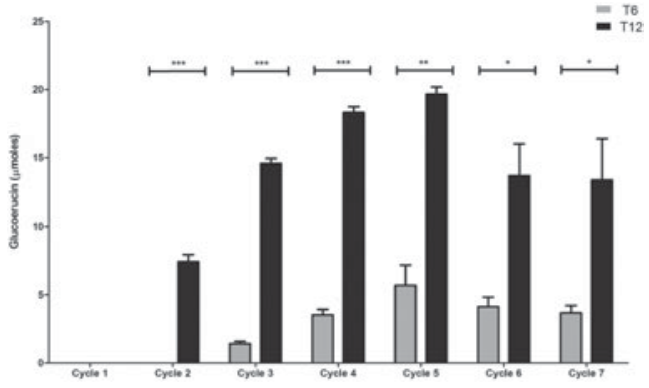

Fig. 2. Increase in glucoerucin across seven 12 -hour cycles.

The data in figure 1 was statistically analysed using one-way ANOVA, and the data in figure 2 was statistically analysed using paired student's $t$-test (two-way): ${ }^{*} p \leqslant 0.05,{ }^{* *} p \leqslant 0.01,{ }^{* * *} p \leqslant 0.001 .16 \mathrm{~S}$ rDNA analysis indicated that the Firmicutes dominated the bacterial community for all seven cycles, and principal coordinates of analysis suggest that the profile of the bacterial community changed twice. The first shift occurred during the first four 12 hour cycles, and this was followed by a further shift, independent of the first, across the last three cycles. The results indicate that the human faecal bacteria utilized the glucoraphanin by reducing it to glucoerucin, and the composition of the bacterial community changed twice in a different manner to one another over seven 12-hour cycles of culturing.

The sample was obtained from a human intervention study that was conducted according to the guidelines laid down in the Declaration of Helsinki (HREC 12/EE/0483).

1. Talalay P \& Zhang Y (1996) Biochem Soc T 24, 806-810.

2. Zhang X, Shu X, Xiang Y et al. (2011) Am Soc Nutrition 94, 240-246.

3. Zhang Z, Ober JA \& Kliebenstein DJ (2006) Am Soc Plant Biol 18, 1524-1536. 